University of Nebraska - Lincoln

DigitalCommons@University of Nebraska - Lincoln

$11-24-2010$

Potential barrier effects in two-photon ionization processes

Liang-Wen Pi

University of Nebraska-Lincoln

Anthony F. Starace

University of Nebraska-Lincoln, astarace1@unl.edu

Follow this and additional works at: https://digitalcommons.unl.edu/physicsstarace

Part of the Physics Commons

$\mathrm{Pi}$, Liang-Wen and Starace, Anthony F., "Potential barrier effects in two-photon ionization processes" (2010). Anthony F. Starace Publications. 177.

https://digitalcommons.unl.edu/physicsstarace/177

This Article is brought to you for free and open access by the Research Papers in Physics and Astronomy at DigitalCommons@University of Nebraska - Lincoln. It has been accepted for inclusion in Anthony F. Starace Publications by an authorized administrator of DigitalCommons@University of Nebraska - Lincoln. 


\title{
Potential barrier effects in two-photon ionization processes
}

\author{
Liang-Wen Pi and Anthony F. Starace \\ Department of Physics and Astronomy, The University of Nebraska, Lincoln, Nebraska 68588-0299, USA
}

(Received 2 September 2010; published 24 November 2010)

\begin{abstract}
Potential barriers in the effective radial potential experienced by a photoexcited electron are shown to result in dramatic, resonancelike effects in two-photon ionization processes. In a two-photon ionization process, such potential barriers may affect not only the final state of the electron (as in ordinary photoionization), but also the intermediate-state electron wave packet corresponding to the absorption of one photon. We illustrate these effects for the generalized two-photon cross sections for ionization of $\mathrm{Ar}$ and $\mathrm{Xe}$ within a single-active-electron, central-potential model.
\end{abstract}

DOI: 10.1103/PhysRevA.82.053414

PACS number(s): $32.80 . \mathrm{Rm}, 32.80 . \mathrm{Aa}$

\section{INTRODUCTION}

The development of novel, intense, and tunable freeelectron lasers (FELs), such as, e.g., the Free-Electron Laser in Hamburg (FLASH), the SPring-8 Compact Self-amplified Spontaneous Emission Source (SCSS) Test Accelerator in Japan, and the Linac Coherent Light Source (LCLS) at Stanford, opens a new regime in which nonlinear processes in the extreme ultraviolet (XUV) and $\mathrm{x}$-ray regions can be investigated [1,2]. Recently, interest has focused on multiphoton ionization of $\mathrm{Xe}$ with photons of $93 \mathrm{eV}$ energy [3-7], with some experiments [6,7] indicating the possible influence of the $4 d \rightarrow \varepsilon f$ giant dipole resonance that is well known in single-photon ionization of $\mathrm{Xe}$ (see, e.g., [8-11] and references therein). In single-photon ionization, the giant dipole resonance phenomenon has been interpreted as originating from a potential barrier in a particular oneelectron ionization channel $[8,10]$ or as originating from a many-electron, collective oscillation of the atomic electrons [9,11]. The two interpretations are not inconsistent: While identification of a potential barrier in a single photoionization channel describes the phenomenon qualitatively, inclusion of many-electron correlations is required to achieve quantitative agreement with experiment. Also, interchannel interactions may transmit the effect to other channels. For multiphoton processes in the XUV regime, there are many competing processes leading to multielectron ionization [5]. Using photoelectron spectroscopy, however, two-photon above-threshold ionization (ATI) of the $4 d$ subshell of Xe was measured for 93-eV photons, and evidence of a giant dipole resonance was reported [7].

At present, the approaches most capable of describing multiphoton processes including many-electron correlations are the $R$-matrix Floquet and the time-dependent $R$-matrix methods (see, e.g., Refs. [12,13]). As reported in Ref. [7], for 93-eV photon energy a "not very accurate" $R$-matrix Floquet calculation nevertheless found that the $\mathrm{Xe} 4 d$ subshell two-photon ATI cross section is dominated by the $4 d \rightarrow \varepsilon f \rightarrow \varepsilon^{\prime} g$ channel, where the first step corresponds to the well-known $\mathrm{Xe} 4 d$-subshell giant dipole resonance transition.

In this article, we report model potential results on the frequency dependence of two-photon ionization and ATI cross sections from particular subshells of $\mathrm{Ar}$ and $\mathrm{Xe}$, showing that potential barriers play a large (and in the case of ATI, dominant) role in these new frequency regimes. Our results provide a broader understanding of the resonance feature reported recently in ATI of Xe at the single-photon energy $\hbar \omega=93 \mathrm{eV}$ [7]. A preliminary account of this work and its results was presented earlier [14].

\section{THEORETICAL CONSIDERATIONS AND APPROACH}

The two-photon transition amplitude from initial state $\left|\Psi_{0}\right\rangle$ of energy $E_{0}$ to final state $\left|\Psi_{f}\right\rangle$ of energy $E_{f}$ is

$$
T_{f \leftarrow 0}^{(2)}=\sum_{i}^{\prime}\left\langle\Psi_{f}|D| \Psi_{i}\right\rangle\left(E_{0}+\omega-E_{i}\right)^{-1}\left\langle\Psi_{i}|D| \Psi_{0}\right\rangle,
$$

where the linearly polarized field of frequency $\omega$ is treated using lowest-order perturbation theory, $D$ is the electric dipole operator, $E_{f}=E_{0}+2 \omega,\left|\Psi_{i}\right\rangle$ of energy $E_{i}$ is the $i$ th intermediate state, and the prime indicates both a sum over discretestates $i$ and an integration over continuum intermediatestates $i$. We evaluate Eq. (1) using the Dalgarno-Lewis method [15],

$$
T_{f \leftarrow 0}^{(2)}=\left\langle\Psi_{f}|D| \Lambda\right\rangle,
$$

where $\Lambda$ is the solution of the inhomogeneous equation

$$
\left(E_{1}-H\right) \Lambda=D\left|\Psi_{0}\right\rangle,
$$

where $E_{1} \equiv E_{0}+\omega$ and $H$ is the $N$-electron, nonrelativistic model Hamiltonian,

$$
H=\sum_{i=1}^{N}\left[-(1 / 2) \nabla_{i}^{2}+V\left(r_{i}\right)\right]
$$

where $V(r)$ is the Herman-Skillman central potential [16], which includes the electron-nucleus interaction and which accounts for electron-electron interactions in an average way. In the single-active-electron approximation, Eq. (3) and the usual Schrödinger equation for $\left|\Psi_{f}\right\rangle$ reduce to equations for the active electron's intermediate and final radial functions, $\lambda_{\varepsilon_{1} \ell}(r)$ and $\psi_{\varepsilon_{f} \ell^{\prime}}(r)$, respectively, where $\varepsilon_{1} \equiv \varepsilon_{0}+\omega, \varepsilon_{f} \equiv \varepsilon_{0}+2 \omega$, and $\varepsilon_{0}$ is the active electron's initial orbital energy. For $\varepsilon_{1}<0, \lambda_{\varepsilon_{1} \ell}(r) \rightarrow 0$ when $r \rightarrow \infty$; for $\varepsilon_{1}>0, \lambda_{\varepsilon_{1} \ell}(r)$ satisfies outgoing wave boundary conditions $[17,18]$ and in this ATI case, free-free dipole matrix elements (2) are evaluated using the complex coordinate rotation method of Ref. [19]. We employ atomic units. 


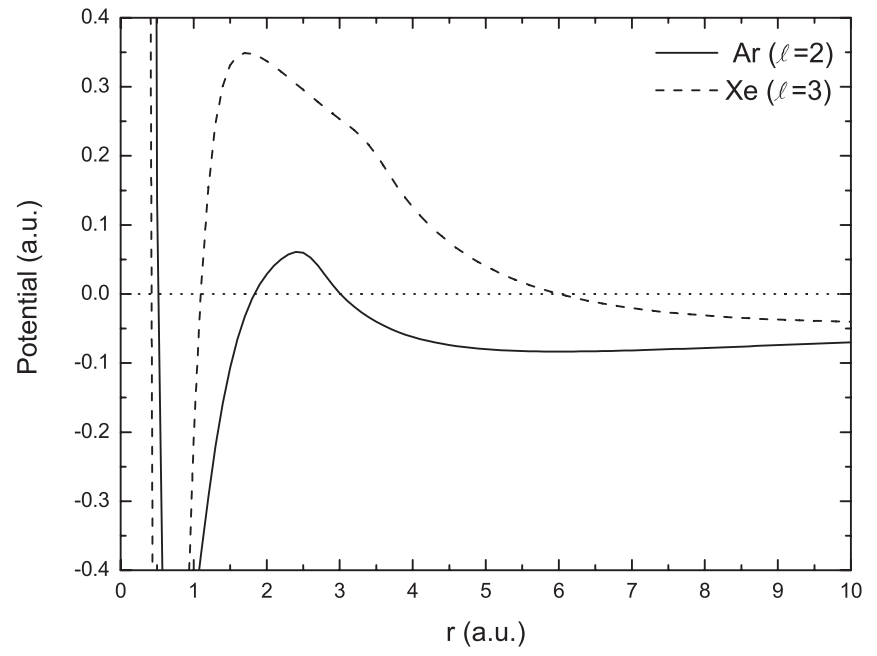

FIG. 1. Effective radial potentials for electrons having orbital angular momentum $\ell=2$ in $\mathrm{Ar}$ and $\ell=3$ in Xe.

Potential barriers may occur in the effective radial potentials, $V_{\text {eff }}(r)=\ell(\ell+1) / 2 r^{2}+V(r)$, that result from reducing Eq. (3) to a radial equation for the active electron. For pure Coulomb potentials, as for the $\mathrm{H}$ atom, $V_{\text {eff }}(r)$ never has a barrier, no matter how large the orbital angular momentum $\ell$. However, for $\ell=2,3$ in heavier atoms, $V_{\text {eff }}(r)$ may have a potential barrier with height $<1$ a.u., typically for $1 \lesssim r \lesssim$ 4 a.u. (cf. Fig. 17 in [8]). Such positive energy barriers stem from the electron-electron repulsion and the centrifugal barrier being greater than the nuclear attraction for radii in the region of the outer subshells of heavier atoms. As examples, Fig. 1 shows the effective potentials for $\operatorname{Ar}(\ell=2)$ and $\mathrm{Xe}(\ell=3)$ with barrier heights of 0.07 and 0.35 a.u., respectively. The barrier results in a double-well effective potential such that ionization from a bound state located in the vicinity of the inner well becomes maximal only for an excited electron energy comparable to the height of the barrier (because continuum states with energies below the barrier height occupy primarily the outer well).

\section{RESULTS AND DISCUSSION}

\section{A. Intermediate-state potential barrier effects}

Consider first the two-photon single ionization of the $2 p$ or $3 p$ subshell of Ar. Each has three channels [20],

$$
\begin{aligned}
\operatorname{Ar} n p^{6}\left({ }^{1} S\right) & +2 \gamma \rightarrow \operatorname{Ar}^{+} n p^{5}\left({ }^{2} P\right) \varepsilon p\left({ }^{1} S\right) \\
& \rightarrow \operatorname{Ar}^{+} n p^{5}\left({ }^{2} P\right) \varepsilon p\left({ }^{1} D\right) \\
& \rightarrow \operatorname{Ar}^{+} n p^{5}\left({ }^{2} P\right) \varepsilon f\left({ }^{1} D\right)
\end{aligned}
$$

For linearly polarized light, the generalized two-photon total cross section, $\sigma$, may be defined in terms of the transition amplitudes $X_{\varepsilon l}^{L M}$ for each of these channels [20]:

$$
\sigma=8 \pi^{3} \alpha^{2} \omega^{2}\left(\left|X_{\varepsilon p}^{00}\right|^{2}+\left|X_{\varepsilon p}^{20}\right|^{2}+\left|X_{\varepsilon f}^{20}\right|^{2}\right),
$$

where $\alpha$ is the fine structure constant, $\omega$ is the photon energy, $L$ is the total orbital angular momentum of the final state, $M=0$ is its projection on the polarization axis, and $\varepsilon$ and $l$ are the energy and orbital angular momentum of the ionized electron.
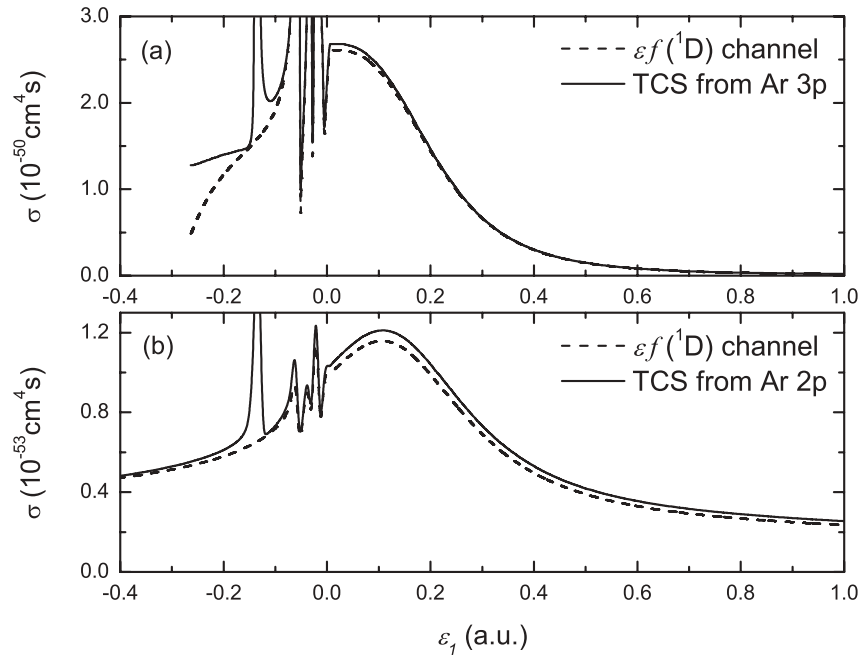

FIG. 2. Solid Lines: Generalized two-photon total cross section (TCS) for ionization from (a) the Ar $3 p$ and (b) the Ar $2 p$ subshells. Dashed Lines: TCS contribution of the $\varepsilon f\left({ }^{1} D\right)$ channel [cf. Eq. (7)]. The one-electron energy $\varepsilon_{1} \equiv \varepsilon_{n p}+\omega$, where $\varepsilon_{n p}$ is the $n p$-orbital energy and $\omega$ is the photon energy.

In the single-active-electron approximation, the amplitudes $X_{\varepsilon l}^{L M}$ reduce to

$$
X_{\varepsilon l}^{L M}=i^{-l} \exp \left(-i \sigma_{l}-i \delta_{l}\right) 3^{-1 / 2}\left[\begin{array}{ccc}
L & 1 & 1 \\
M & 0 & 0
\end{array}\right] T_{f \leftarrow 0}^{(2)},
$$

where $\sigma_{l}$ is the Coulomb phase, $\delta_{l}$ is the phase shift with respect to a Coulomb wave, and $T_{f \leftarrow 0}^{(2)}$, defined by Eq. (1), is calculated using Eq. (2). For details, see Refs. [20,21].

Figure 2 shows the generalized two-photon total cross section, $\sigma$ [cf. Eq. (8)], for ionization of the $\operatorname{Ar} 2 p$ and $3 p$ subshells plotted vs the energy $\varepsilon_{1} \equiv \varepsilon_{n p}+\omega$ of the active electron's intermediate-state wave packet, $\lambda_{\varepsilon_{1} \ell}(r)$ [cf. Eq. (3)]. Thus, $\varepsilon_{1}<0$ implies two-photon ionization, while $\varepsilon_{1}>0$ implies ATI. Although $\sigma$ for the $3 p$ subshell is $10^{3}$ times larger than that for the $2 p$ subshell, the resonancelike shapes of $\sigma$ for the two subshells are remarkably similar: Both have Rydberg resonances below $\varepsilon_{1}=0$, a maximum just above, followed by a smooth, broad decrease. The maximum occurs at $\varepsilon_{1}=0.05$ a.u. for $\operatorname{Ar} 3 p$ and 0.1 a.u. for Ar $2 p$, both of which are close to the height of the potential barrier, 0.07 a.u., for $\operatorname{Ar}(\ell=2)$, shown in Fig. 1. The broad resonance in $\sigma$ for both subshells extends into the below-threshold region (i.e., $\varepsilon_{1}<0$ ) owing to the low height of the potential barrier for $\operatorname{Ar}(\ell=2)$ so that the resonancelike maximum occurs close to $\varepsilon_{1}=0$. Our calculations show that the $\varepsilon f$ final-state channel (7) provides more than $95 \%$ of the value of $\sigma$, while the other two channels are highly suppressed. Channel (7), in addition to having an intermediate $d$ state that experiences a potential barrier, involves also a sequence of transitions in which both the active electron's orbital angular momentum and the system's total angular momentum increase with each photon absorption; that is, $p\left({ }^{1} S\right) \rightarrow d\left({ }^{1} P\right) \rightarrow f\left({ }^{1} D\right)$. The other two channels, (5) and (6), also have contributions from intermediate $d$ states that experience the potential barrier, but upon absorbing the second photon the active electron's orbital angular momentum 


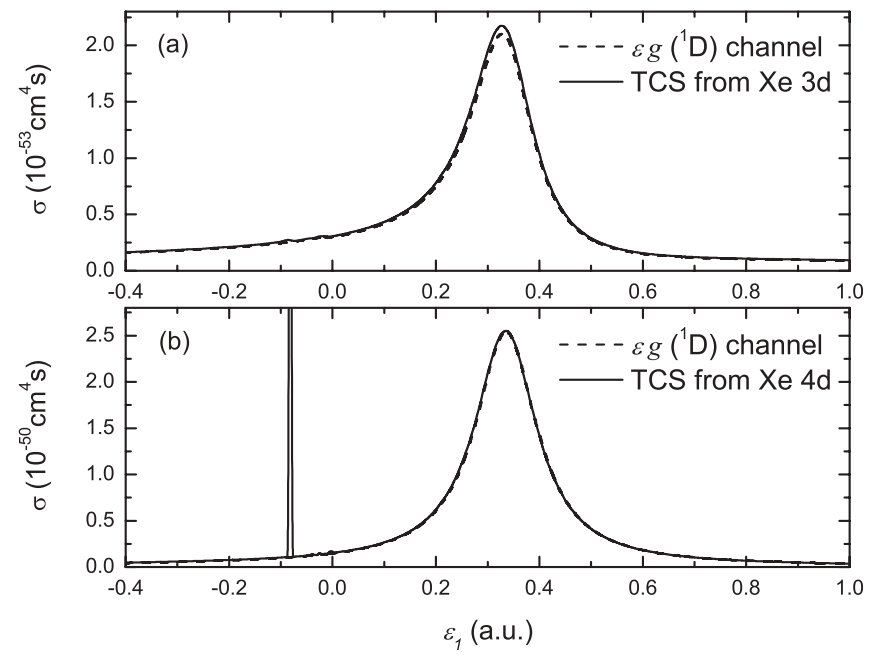

FIG. 3. Solid Lines: Generalized two-photon TCS for ionization from (a) the Xe $3 d$ and (b) the Xe $4 d$ subshells. Dashed Lines: TCS contribution of the $\varepsilon g\left({ }^{1} D\right)$ channel [cf. Eq. (13)]. The one-electron energy $\varepsilon_{1} \equiv \varepsilon_{n d}+\omega$, where $\varepsilon_{n d}$ is the $n d$-orbital energy and $\omega$ is the photon energy.

decreases and, in the case of (5), so does the total orbital angular momentum.

Two-photon single ionization of the $3 d$ or $4 d$ subshell of $\mathrm{Xe}$ is a bit more complicated, as each has four channels:

$$
\begin{aligned}
\mathrm{Xe} n d^{10}( & \left({ }^{1} S\right)+2 \gamma \rightarrow \mathrm{Xe}^{+} n d^{9}\left({ }^{2} D\right) \varepsilon s\left({ }^{1} D\right) \\
& \rightarrow \mathrm{Xe}^{+} n d^{9}\left({ }^{2} D\right) \varepsilon d\left({ }^{1} S\right) \\
& \rightarrow \mathrm{Xe}^{+} n d^{9}\left({ }^{2} D\right) \varepsilon d\left({ }^{1} D\right) \\
& \rightarrow \mathrm{Xe}^{+} n d^{9}\left({ }^{2} D\right) \varepsilon g\left({ }^{1} D\right)
\end{aligned}
$$

Figure 3 shows the generalized two-photon total cross section, $\sigma$, for ionization of the Xe $3 d$ and $4 d$ subshells plotted vs the energy $\varepsilon_{1} \equiv \varepsilon_{n d}+\omega$ of the active electron's intermediatestate wave packet, $\lambda_{\varepsilon_{1} \ell}(r)$ [cf. Eq. (3)]. In each case, the resonance maximum is at $\sim 0.34$ a.u., which is very close to the $\mathrm{Xe}(\ell=3)$ potential barrier height, 0.35 a.u., shown in Fig. 1. Our calculations show that the $\varepsilon g$ final-state channel (13) provides more than $95 \%$ of the value of $\sigma$, while the other three channels are highly suppressed, for reasons similar to those adduced for Ar. In contrast to results for Ar, there is a notable absence of Rydberg structure for $\varepsilon_{1}<0$. This stems largely from the large potential barrier in Xe seen by the $\ell=3$ intermediate states, which results in most Rydberg $f$ states occupying the outer well (cf. Fig. 1) so that they have little overlap with the $n d$-initial states. [The single Rydberg resonance shown in Fig. 3(b) just above $\varepsilon_{1}=-0.1$ a.u. is due to a $4 d \rightarrow 6 p$ transition.]

In order to elucidate the origin of the giant resonance seen in Fig. 3(b), we examine in more detail the dominant channel (13), which involves the two dipole transitions, $4 d \rightarrow \varepsilon_{1} f \rightarrow \varepsilon_{f} g$ (where $\left.\varepsilon_{f}=\varepsilon_{4 d}+2 \omega\right)$. The transition rate for this channel is proportional to the radial dipole matrix element $\left\langle\psi_{\varepsilon_{f} g}|r| \lambda_{\varepsilon_{1} f}\right\rangle$ [cf. Eq. (2)]. In Fig. 4 we plot the final-state radial wave function, $\psi_{\varepsilon_{f} g}(r)$, and the amplitude of the complex intermediate-state electron wave packet, $\left|\lambda_{\varepsilon_{1} f}(r)\right|$, as functions of the radial coordinate $r$ for three different photon energies, corresponding to energies $\varepsilon_{1}$ below, at, and above

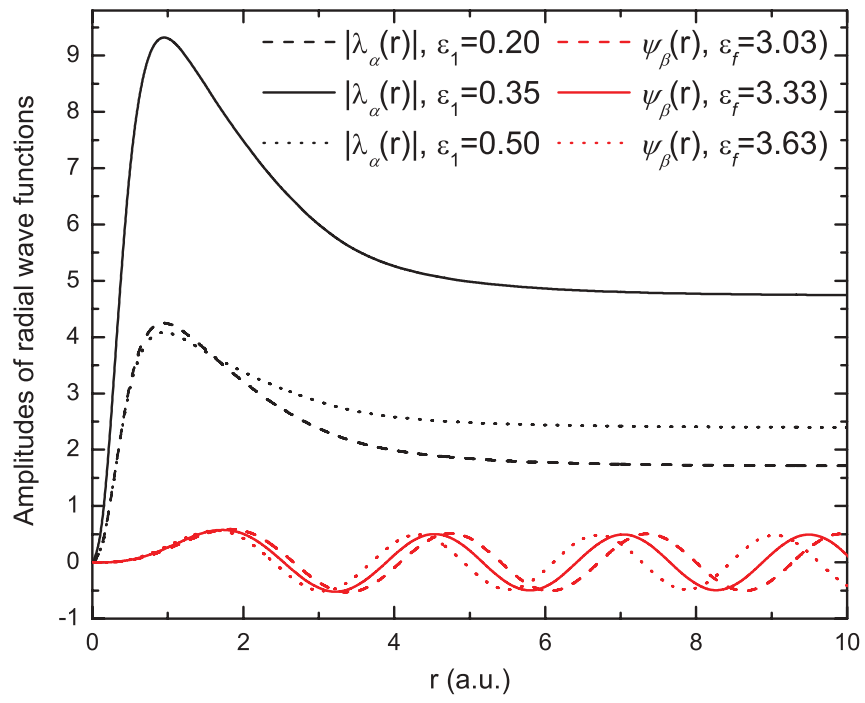

FIG. 4. (Color online) Upper three (black) curves: Amplitude of the complex intermediate-state radial wave packet, $\left|\lambda_{\alpha}(r)\right| \equiv$ $\left|\lambda_{\varepsilon_{1} f}(r)\right|$, for three energies $\varepsilon_{1} \equiv \varepsilon_{4 d}+\omega$ below, at, and above the resonance maximum in Fig. 3(b). Lower three (red) curves: Corresponding final-state radial wave functions, $\psi_{\beta}(r) \equiv \psi_{\varepsilon_{f} g}(r)$, where $\varepsilon_{f} \equiv \varepsilon_{4 d}+2 \omega$.

the resonance maximum in Fig. 3(b). One observes that the final-state radial wave functions, $\psi_{\varepsilon_{f} g}(r)$, have nearly the same amplitudes. However, the amplitudes of the intermediate radial wave packets, $\left|\lambda_{\varepsilon_{1} f}(r)\right|$, change dramatically: At the energy of the resonance maximum, there is a more than factor of two increase in amplitude compared to the energies below and above the maximum. The resonance maximum is thus due to a transitory trapping of the intermediate-state electron wave packet by the potential barrier at energies in the vicinity of the barrier height.

\section{B. Final-state potential barrier effects}

In ordinary photoionization, it is the final-state electron that probes the potential barriers shown in Fig. 1. In a two-photon ionization process, the final state may also probe these barriers, but (owing to dipole selection rules) the active electron must originate from an initial state with the same parity as the final state. We illustrate this by examining the two-photon ionization of the Ar $3 s$ subshell, for which there are only two channels:

$$
\begin{gathered}
\operatorname{Ar} 3 s^{2}\left({ }^{1} S\right)+2 \gamma \rightarrow \operatorname{Ar}^{+} 3 s^{1}\left({ }^{2} S\right) \varepsilon s\left({ }^{1} S\right), \\
\rightarrow \operatorname{Ar}^{+} 3 s^{1}\left({ }^{2} S\right) \varepsilon d\left({ }^{1} D\right) .
\end{gathered}
$$

Our results for the generalized two-photon ionization cross section, both total and for each of the channels (14) and (15), are shown in Fig. 5. Unlike in the four ATI cases shown in Figs. 2 and 3, in each of which there is only one dominant channel, for this case in which the final-state electron probes the potential barrier the contributions of the two allowed channels are comparable. The total cross section has a resonance at 0.08 a.u., which is close to the barrier height for $\operatorname{Ar}(\ell=2)$ (cf. Fig. 1) experienced by the electron in the $\varepsilon d$ final-state channel (15).

An unexpected feature in Fig. 5 is the Cooper minimum near $\varepsilon_{f}=0.3$ a.u., originating from a zero in the partial 


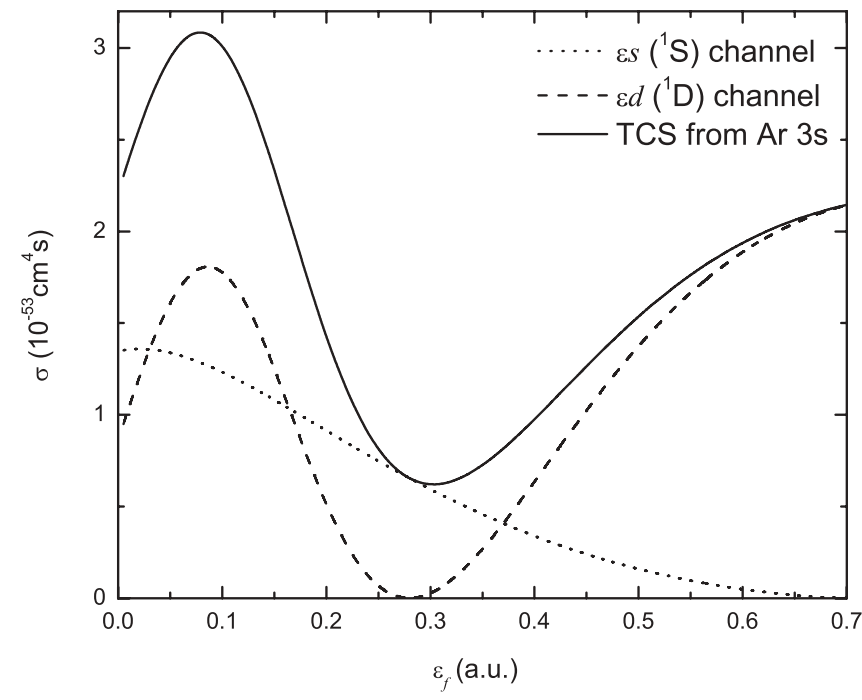

FIG. 5. Generalized total and partial two-photon ionization cross sections for the $\operatorname{Ar} 3 s$ subshell. $\varepsilon_{f} \equiv \varepsilon_{3 s}+2 \omega$ is the ionized electron's energy, where $\varepsilon_{3 s}$ is the $3 s$ orbital energy.

cross section for the $\varepsilon d$ channel (15). Cooper minima have been investigated systematically in single-photon ionization processes (see, e.g., Refs. [22-24]), but have not been investigated for multiphoton processes. Our calculations show that as the energy of the final state varies across the height of the potential barrier, its phase shift changes dramatically, so that the overlap of the intermediate-state wave packet and the final state changes [cf. Eq. (2)], leading to a sign change in the radial part of the transition amplitude. However, rules of thumb for predicting when and where such minima occur requires more detailed theoretical and numerical study.

\section{CONCLUSIONS}

In conclusion, our results for two-photon ionization of various subshells of $\mathrm{Ar}$ and $\mathrm{Xe}$ (as well as other results, not shown, for $\mathrm{Au}, \mathrm{Ag}, \mathrm{Cu}$, and $\mathrm{Hg}$ ) indicate that potential barrier effects may be general features of multiphoton ionization processes. (We are currently investigating three-photon processes to confirm this expectation.) For the two-photon cases considered here, the most dramatic results occur in ATI processes when the intermediate-state electron wave packet having a high orbital angular momentum probes the corresponding potential barrier. Far from being merely a convenient way to sum over a complete set of intermediate states, the intermediate-state electron wave packet obtained by solving Eq. (3) may be used to obtain insight into key features of two-photon processes. In the two-photon ionization case, in which the final-state electron probes the potential barrier, Cooper minima originating from rapid changes in the final-state phase shift are also likely to be common features, but require more systematic investigations. We emphasize that while electron correlations will alter the present results quantitatively, the dramatic resonancelike enhancements that we predict represent a qualitatively correct guide for both experiments and also for more detailed theoretical calculations.

\section{ACKNOWLEDGMENTS}

This work is supported in part by the Department of Energy, Office of Science, Division of Chemical Sciences, Geosciences, and Biosciences, under Grant No. DE-FG03-96ER14646, and by National Science Foundation Grant No. PHY05-51164. A.F.S. gratefully acknowledges the hospitality of the Kavli Institute for Theoretical Physics, University of California at Santa Barbara, where part of this article was prepared.
[1] J. Feldhaus, J. Arthur, and J. B. Hastings, J. Phys. B 38, S799 (2005).

[2] N. Berrah et al., J. Mod. Opt. 57, 1015 (2010).

[3] A. A. Sorokin, S. V. Bobashev, T. Feigl, K. Tiedtke, H. Wabnitz, and M. Richter, Phys. Rev. Lett. 99, 213002 (2007).

[4] M. Richter, S. V. Bobashev, A. A. Sorokin, and K. Tiedtke, Appl. Phys. A 92, 473 (2008).

[5] M. G. Makris, P. Lambropoulos, and A. Mihelič, Phys. Rev. Lett. 102, 033002 (2009).

[6] M. Richter, M. Y. Amusia, S. V. Bobashev, T. Feigl, P. N. Juranic, M. Martins, A. A. Sorokin, and K. Tiedtke, Phys. Rev. Lett. 102, 163002 (2009).

[7] V. Richardson et al., Phys. Rev. Lett. 105, 013001 (2010).

[8] U. Fano and J. W. Cooper, Rev. Mod. Phys. 40, 441 (1968), Sec. 4.4.

[9] M. Ya. Amusia and N. A. Cherepkov, Case Stud. At. Phys. 5, 47 (1975).

[10] A. F. Starace, in Handbuch der Physik, edited by W. Mehlhorn (Springer-Verlag, Berlin, 1982), Vol. 31, p. 1.
[11] M. Ya. Amusia and J. P. Connerade, Rep. Prog. Phys. 63, 41 (2000).

[12] H. W. van der Hart, Phys. Rev. A 73, 023417 (2006).

[13] H. W. van der Hart, M. A. Lysaght, and P. G. Burke, Phys. Rev. A 76, 043405 (2007).

[14] L. W. Pi and A. F. Starace, Bull. Am. Phys. Soc. 55(5), 156 (2010), Abstract T1 34.

[15] A. Dalgarno and J. T. Lewis, Proc. R. Soc. A 233, 70 (1955).

[16] F. Herman and S. Skillman, Atomic Structure Calculations (Prentice-Hall, Englewood Cliffs, NJ, 1963).

[17] F. Robicheaux and B. Gao, Phys. Rev. A 47, 2904 (1993).

[18] M. Marinescu, H. R. Sadeghpour, and A. Dalgarno, J. Opt. Soc. Am. B 10, 988 (1993).

[19] B. Gao and A. F. Starace, Comput. Phys. 1, 70 (1987).

[20] C. Pan and A. F. Starace, Phys. Rev. A 44, 324 (1991).

[21] C. Pan, B. Gao, and A. F. Starace, Phys. Rev. A 41, 6271 (1990).

[22] J. W. Cooper, Phys. Rev. 128, 681 (1962).

[23] S. T. Manson, Phys. Rev. A 31, 3698 (1985).

[24] R. H. Pratt, R. Y. Yin, and X. Liang, Phys. Rev. A 35, 1450(R) (1987). 